\title{
Methodology for Evaluating Multimodal Biometric Authentication on Mobile Devices
}

\author{
Claudia de Andrade Tambascia, Robson Eudes Duarte, \\ and Ewerton Martins Menezes \\ Telecommunications Research and Development Center \\ Campinas, São Paulo, Brazil \\ \{claudiat, robsond, emenezes\} @ cpqd. com.br
}

\begin{abstract}
Biometric authentication systems are an important component in more sensitive applications, since, in conjunction with the use of a mobile device, enable so-called "multifactor authentication", which involves something the user knows (a secret, e.g. password or PIN), something he has (mobile device) and something that he is (a biometric trait). This paper aims to present usability recommendations to be followed during the implementation of applications or services for mobile devices. These recommendations will be presented taking into account international standards and standards already used in other applications and devices. As a result it is expected a set of specific recommendations for the technology that will be adopted in the context of BIOMODAL project.
\end{abstract}

Keywords: Biometric authentication systems, Usability recommendations for mobile devices, Multifactor authentication.

\section{Introduction}

When thinking about mobile devices and their applications, it becomes possible to say that today the mobility aspects are increasingly common and necessary for society as a whole. Users of this type of technology have a different usage experience of desktop and notebook users, which demands that computer systems developed for mobile technology take into account some specific features to ensure that usability requirements are met. When these requirements are not met, it is often possible to associate users non satisfaction with aspects related to:

- Hardware devices with a design non appropriated for use;

- Information and content with none customization to the mobile environment;

- A lot of information presented on the screen, making the location desired by the user's option very difficult;

- Content loading time inappropriate, due to slow connection speeds, stopping interactions [1].

The purpose of this paper is to present usability recommendations that should be followed during applications and services development for mobile devices, and contributing to ensure the quality of experience of end-users. These recommendations 
are based on international norms and standards and will be adapted according to mobile technology that is assumed and the prototype which will be developed in the context of BIOMODAL project, thus allowing the development of a methodology for usability evaluation to be applied on these applications and services.

\section{Definitions}

The term Usability, defined in [2], is related to the measure by which a product can be used by specified users to achieve specific goals with effectiveness, efficiency and satisfaction in a context of use. Among the specific objectives to be measured and achieved are considered aspects that allow the user to reach his/her goal, taking into account the necessary effort to ensure that this goal is met.

Under the BIOMODAL project it was defined, in the first time, the applications development for Android platform, because is an open source and it's growing in the global market. These technologies minimize testing costs and requirements definition that must be followed in the applications development to be available.

In the following section it will be presented usability recommendations used in this specific project for mobile devices, as well as standards considered for this technology. Such information will be used during the creation of Mobile Devices Recommendations Guide (MDRG).

\section{State of Art}

Despite the BIOMODAL project considers the use of mobile devices like iPhone and Android operating system, usability recommendations were searched for mobile applications embracing also other technologies and it was found that many of these recommendations can be considered universal, and others may be completely dependent on application-specific requirements context. This paper will present some recommendations identified for different devices and recommendations which should be fully considered in applications development for BIOMODAL project.

\subsection{Applications for iPhone}

Recommendations for iPhone were defined in a guide structured into two parts. The first one describes the iPhone OS environment and types of software that can be developed, covering also fundamentals of interface design and describing how to apply these principles in applications iPhone design [3]. The second part describes several interfaces elements available in the iPhone SDK and how to use them when building an application.

An important aspect outlined in this guide is related much more to the differences between developing applications for mobile devices and computers and how it impacts on design decisions. These differences are related to the small size of the screen, limited memory, and visualization of one screen at a time and, in the iPhone specific case, running only one application at a time. In terms of implementation, 
native applications must appreciate the ease, simplicity, responsiveness and esthetic interface appearance. For this reason it was defined styles provided by the application based on visual characteristics and behavioral data model and user experience where productivity applications must keep the user focused on the task and information, usually by putting the information in a hierarchical manner. It can also offer customization options, utility applications should require minimal user input, be visually attractive and flexible for different types of content on the other hand immersion applications should occupy the full screen and offer a customized interface, with a visually rich environment focused on content and user experience, being used mostly for fun.

In the BIOMODAL project context development will be held within productivity applications mostly though it will also consider utility applications. Another important aspect to be considered relates to porting applications from desktop to mobile platform. In this case, it is important to consider the different expectations and context of use by the user of the application, because often the use of the iPhone is done in environments full of distractions, with brief task execution and does not require total attention and may be a good practice to focus on the application iPhone in features that cover the greatest number of users.

The features to consider on an interface project for iPhone are elements like metaphors, direct manipulation, choice, feedback mechanisms, user control, aesthetic integrity, intelligibility, organization, text input, informational texts, primary tasks, targets, and effective communication, and for each one, respective recommendations were defined and some of them will be used in BIOMODAL project.

The iPhone recommendations presents a constant concern in maintaining the native operating system standards in applications developed for this environment, such as color scheme, interface elements and their behaviors so the user could transfer the knowledge acquired with the iPhone OS interface for its applications. This might be a recommendation to be followed during the BIOMODAL project.

\subsection{Applications for BlackBerry Smartphones}

A recommendation guide defined to applications developers for BlackBerry smartphone platform gathers specific tips and good practice design interfaces [4] with great importance for BIOMODAL project.

Applications developed for BlackBerry platform must provide a balance between the best user experience possible and battery consumption level. Taking into consideration the main differences between mobile devices and traditional computers, it must be also listed factors as small screens, processor with lower speed, low memory, battery charging time and the limitation of only one screen be displayed at a time as limiting factors in the development of applications for this type of device.

How the user interacts with the mobile application is also different than the way of interaction on computers applications. In this case, the efficiency and speed with which the user performs the tasks in this environment are only reached understanding user navigation model and providing mechanisms for this kind of interaction happens as quickly and easily as possible. 
The features to be considered for this item on a interface project for Smartphones include elements such as navigation, interface components, focus on the task, menu actions, undo function, information presentation, touch screen, keyboard, cursor, application screens, banners, dialog boxes, progress indicators, information and images, menus, fonts, conventions of writing, icons, sounds, location, design of the interface and colors, with their respective recommendations and were also considered in BIOMODAL project too.

\subsection{Applications for Android}

Developing applications for Android is supported by a dedicated portal where developers found articles about operating system compatibility with different devices and design recommendations focused on performance, responsiveness and treatment of interruptions.

As the Android operating system runs on a variety of devices, compatibility is a very important requirement in their applications. One of the ways to promote compatibility is filtering applications to be installed on a given device according to the hardware characteristics, such as gifts, compass, accelerometer, camera, touchscreen, among others. In addition, another point to be considered on how to increase compatibility is to support the screens of different sizes and resolutions. It is possible, in a file called manifest of each application, set attributes on which sizes and resolutions are supported, where the screens are categorized into large, normal, or small relative to its size, density and high, medium or low.

The features to be considered on a project interface for the Android operating system take into account elements like application screen, presentation, interruption of activity, back key, home key, menus and commands, with their respective recommendations.

Due to restrictions on mobile devices, this type of applications should have a particular concern in relation to the performance, responsiveness and disruptions suffered by the user. In Best Practices is given a detailed approach on how to achieve these goals, in order to keep its code always efficient. It is important to note that the application should not run tasks that the user didn't need nor allocate memory when you can avoid.

\subsection{Applications for Other Mobile Devices}

Studies conducted by researchers at Americans in [5] made comparisons of limitations and features of mobile devices interfaces developed with desktop interfaces, using the existing interface recommendations for desktop as a starting point for the formulation of mobile devices specific recommendations, presented as rules that are used as the basis of that study and among the eight initial recommendations. Four of them are kept fully and the remaining is adapted to the mobile context [6]. The key features for the mobile device interface design are elements such as shortcuts, feedback, dialogues, sequential actions, control, consistency, rollback actions, errors, memorization, multiple and 
dynamic contexts, size of devices, modes of interaction, storage and retrieval, interaction top-down, customization, and aesthetics, with their respective recommendations.

From all the studies conducted, researchers have made important discoveries of usability, among which we can mention that in mobile applications the tasks performed by the users should be appropriate to the time and cognitive resources employed in its realization. And the design must be done so that the user can transfer the knowledge acquired with the applications that he/her has already touched.

Due to limited data bandwidth, content intended for mobile devices must be smaller than the normal site content and must not require very large downloads, since losses are very frequent. While vertical screen scrolling is not wanted, scrolling the horizontal should be avoided at all cost because it generates serious flaws in mobile functionality.

\subsection{Usability and Biometrics}

Aspects related to ensuring successful biometric systems are connected directly with usability-related factors, such as the National Institute of Standards and Technology (NIST). The documentation provided is intended for designers and developers of biometric technologies, describing the process of user centered development and how to incorporate this process can improve the effectiveness, efficiency and user satisfaction in biometric systems.

Currently aspects of users (age, gender, experience, abilities and limitations) have been disregarded in design of biometric systems, with main focus on performance, functionality, reliability and accuracy of system, setting aside the user and his/her influence on the system. In fact, the user is a key piece on biometric process because only with user's acceptance any biometric technology can evolve.

The features to be considered on this item for a project on biometric applications are elements such as effectiveness, efficiency, customer satisfaction, ease of learning and ease of memorization, with their respective recommendations.

\section{Aspects Related to the Biometric Systems Development}

Although usability and accessibility recommendations are listed in the context of development of biometric systems, to improve the ability of end-users in the use of a product effectively and efficiently, the process of user centered design must be considered to be of great importance.

This process is considered interactive with a lifecycle that is composed of four main phases and which are known to cause measurable impacts on usability and ease of use of biometric systems.

The four main stages of the process are: definition of the usage context, determining the target users and organizational requirements, development of design solution and assessments implementation. Figure 1 illustrates the defined phases. 


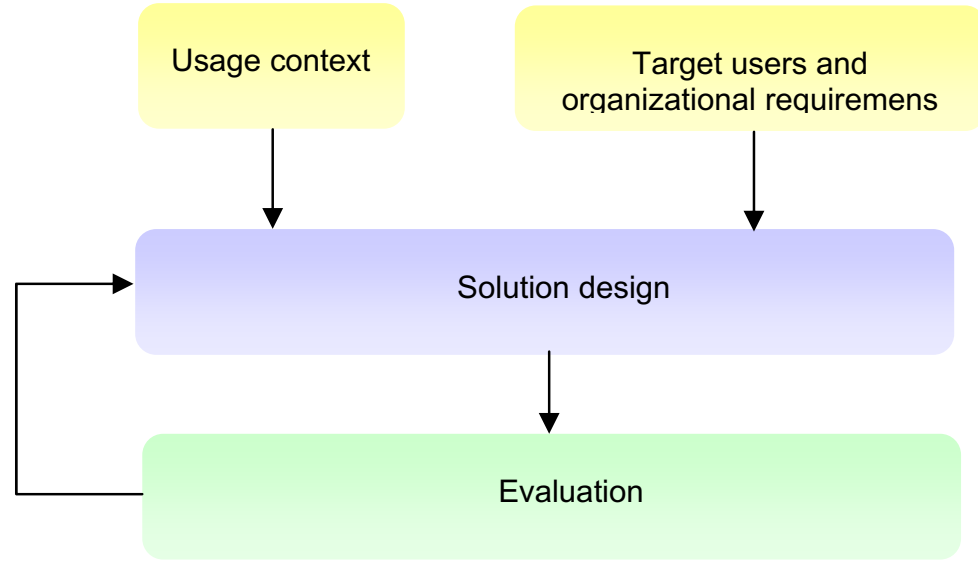

Fig. 1. Phases for biometric systems development

The definition of usage context involves not only the user's context, but also the business environment in which the biometric system is developed, the operating environment where the system will be used and the social environment where it will be implemented. Understanding and sorting system users is an important task in this definition, because it facilitates the development of a system that meets the needs and expectations of users. One way to achieve this goal is to define characteristics of the end users and how they can influence the final design of the system. These characteristics are summarized in Table 1.

Table 1. End user features

\begin{tabular}{ll}
\hline Features & Questions to consider \\
\hline Age & $\begin{array}{l}\text { Older users need larger texts or sounds higher tips. Younger } \\
\text { users are not necessarily more successful }\end{array}$ \\
\hline Genre & Genre may or may not be more successful than another. \\
\hline Anthropomorphic & User height can affect his/her ability to use the system. \\
\hline $\begin{array}{l}\text { Ethnicity, nationality, language } \\
\text { and culture }\end{array}$ & $\begin{array}{l}\text { Without a common language can be no impact on the } \\
\text { design of feedback and instruction guide. }\end{array}$ \\
\hline Education & $\begin{array}{l}\text { Education can affect system usage. Biometric systems } \\
\text { should cover users with little or no ability to read. }\end{array}$ \\
\hline $\begin{array}{l}\text { Experience and knowledge } \\
\text { level }\end{array}$ & $\begin{array}{l}\text { Novice user needs differ from the frequent or regular user } \\
\text { needs. }\end{array}$ \\
\hline Accessibility attributes & $\begin{array}{l}\text { Design requirements should be considered to make the } \\
\text { system accessible universally. }\end{array}$ \\
\hline
\end{tabular}

In addition to the aspects related to the user, environmental factors may influence or produce any effect that can directly influence the design of biometric systems. These factors are described in reference Table 2 . 
Table 2. Environmental factors influence in biometric systems

\begin{tabular}{ll}
\hline Features & Potential impact \\
\hline Location & $\begin{array}{l}\text { Physical environment in which the biometric system is } \\
\text { affects or influences the design. }\end{array}$ \\
\hline Device location & $\begin{array}{l}\text { Device's location affects the user's ability to use or access } \\
\text { the system. }\end{array}$ \\
\hline Temperature and humidity & $\begin{array}{l}\text { External environment affects the system performance and } \\
\text { temperature and humidity affects the capture of a high- } \\
\text { quality sample. }\end{array}$ \\
\hline $\begin{array}{l}\text { Ethnicity, nationality, language } \\
\text { and culture }\end{array}$ & $\begin{array}{l}\text { Without a common language there can be no impact on the } \\
\text { design of feedback and instruction guide. }\end{array}$ \\
\hline Lighting & $\begin{array}{l}\text { The light level lighting affects the reading or the visibility } \\
\text { of the graphics device. }\end{array}$ \\
\hline Noise & $\begin{array}{l}\text { The noise level can affect the ability of an individual to } \\
\text { hear tips and information for audio and give a feedback to } \\
\text { the system. }\end{array}$ \\
\hline Instructions & $\begin{array}{l}\text { If there are instructions, icons and sizes of the letters must } \\
\text { be seen as appropriate and understandable. }\end{array}$ \\
\hline Help & $\begin{array}{l}\text { In a given environment, it must be known the better way to } \\
\text { provide feedback, errors and help information. }\end{array}$ \\
\hline
\end{tabular}

With the definition of use context it is also defined tasks the user will do in the system, seeking to prioritize them according to user needs, usage frequency, importance, vulnerability, and if it is doable.

In the solution design phase, the design considerations should not be limited to end users, but should consider the users who will operate the system and analyze the data. The goal of the interaction design is shaping the behavior of the system, without pegging it to an interface, so that system and user can work in partnership to achieve the end goal of the application. For each user action that the system should provide a feedback and to make that possible, a solid design specification is required.

As soon as "what to" and "when to" do are defined in interface design, the main point is how to make the communication between the system and the user. A consensus is that the system should provide feedback in a multitude of ways, just in case something fails or the user does not understand the method chosen. In addition to considering the contents, the interface design process must also consider the most effective way to display it to the user.

Finally the last phase is the evaluation process, an essential part of the process because it provides assurance that the design is being developed correctly, assisting in the identification of problems that still need to be resolved.

Evaluation and design are phases that must be done interactively, where the results obtained with the end users in reviews are incorporated into the design making the system meet as much as possible the needs of users. In the initial stages of design, the assessment should be more qualitative, where impressions and reactions of users are of great importance. In the later stages of development, though, the system must be tested by means of more quantitative usability testing, where the performance when using the system is measured. 


\section{Guide Recommendations for Mobile Devices in the Context of BIOMODAL Project}

Based on research described in section 2 of this paper it's possible to determine usability recommendations that should be considered in the biometric recognition prototype to be developed in BIOMODAL project context.

For this purpose it was defined categories where every aspect to consider is detailed, showing its definition and the associated recommendation, looking to illustrate with possible solutions for development.

This process of categorization and descriptions of the recommendations is done by setting the requirements of the application being developed. Therefore, it is necessary to wait for completion of the project requirements document of BIOMODAL for specific recommendations to guide the implementation. What can be done initially is the division of components that will be treated in the interface and interface elements that will be associated and which recommendations should be followed in the development of the prototype. Table 3 presents the elements which should in principle be treated and their groupings, according to the component under consideration.

Table 3. Components and elements to be considered in the BIOMODAL prototype

\begin{tabular}{ll}
\hline Element & Components \\
\hline Window structure & $\begin{array}{l}\text { Title bar, application area, scroll bar and secondary } \\
\text { window. }\end{array}$ \\
\hline Menu structure & $\begin{array}{l}\text { Menu bar, number of options, drop-down menu and } \\
\text { contextual menu. }\end{array}$ \\
\hline Forms structure & $\begin{array}{l}\text { Structure check, grouping information, separator lines, } \\
\text { input and output groups and commands group. }\end{array}$ \\
\hline Information structure & Information group and encoded information. \\
\hline Driving mechanisms & $\begin{array}{l}\text { Guidance, feedback, status, control, error prevention } \\
\text { and error correction mechanisms, error message and } \\
\text { help engine. }\end{array}$ \\
\hline Presentation & $\begin{array}{l}\text { Used colors, font style, font size, icons and selection or } \\
\text { pointing area. }\end{array}$ \\
\hline Interaction & Browsing interaction model, callback option and \\
steps to run the task.
\end{tabular}

The components and elements defined, associated with its recommendations, will be also used in methodology of validation of applications for mobile devices, and so the recommendations must be clear and well defined, so that developers won't have questions about how to deploy the application.

\section{Conclusion}

Usability recommendations are considered an important requirement in the BIOMODAL project context to guide developers the implementation of services or applications, ensuring the user-friendliness and satisfactory execution of tasks, contributing to the quality of user experience. 
For adequate preparation of the Recommendation Guide, it was necessary to determination the family of devices to be covered by the project, as well as the requirements of prototype being developed, so that in accordance with the Guide, is designed a usability evaluation methodology for applications on mobile devices.

Any system that intends to reach the mobile market has to deal with specific usability issues from the mobile context like small screens, inappropriate design of hardware, problematic input method, inadequate content loading time and the dynamic context of use. We aim mainly to define usability aspects which must be tracked throughout the services and applications development for mobile devices as well as the contribution of these aspects to user's Quality of Experience (QoE) enhancement.

Those usability issues emerge from international standards in the scope of an R\&D project of multimodal biometric authentication for banking services on mobile devices. The methodology proposed should determine how to measure, categorize, identify and observe user's interaction with such services in a usability laboratory. Taking into account that the user's perceptions and expectations of the system are crucial in biometric authentication, it is important not just to improve its performance and effectiveness but also to make it useful in the real world.

To properly run the usability tests defined on the methodology we recommend a lab composed by two distinct rooms. One of them is the test or interaction room, used to host, accommodate and conduct the users who will perform the tasks, equipped with computers, mobiles devices, monitoring cameras and touchscreen monitors. The other is the observation room, an annex building to the test room separated from it by a two-way mirror, used to accommodate the professional team who will follow the tasks execution and manage the tests recording. In order to better organize the usability activities and reach the project HCI goals, the methodology can be customized to the defined test purpose and involves 4 main stages: contextual analysis, test setting, test execution and results analysis and interpretation.

On the contextual analysis the test techniques and the application platform are defined with a range of available options, such as, quali-quantitative evaluations, use of real devices or emulators, simultaneous or consecutive verbalizations and previous heuristic evaluations. The specialists should align the chosen techniques according to the test purposes and platform and for this reason a broad knowledge about the project and its current development status is required.

On the test setting stage it is necessary to determine the number of target users that will perform the test, the tasks which will be included in the test process and define more than one data storage and noting techniques. Moreover it is important to set the test environment so that it would get as near as possible to the real context of use. It is also on this stage that the participants recruiting process happens.

On the test execution stage, a pilot test must take place to ensure that all the previous settings are utterly and properly working. During the test process, it is preferable that only one from the usability team addresses personally to the user to clarify the tasks to be performed, lead and help in case of critical doubt and to start and finish the test. By the beginning of the process, the conductor must explain the test purposes, making it clear that it is not the user who will be evaluated but the developed solution. Annotations must refer the time of relevant events (doubts, confusion, errors) and profile or satisfaction interviews may take place before the test ends. 
Finally, the stage of results analysis and interpretation must allow the whole usability team to review the recordings and recover relevant data which might confirm or disprove formerly written hypothesis. In this stage a report must be made, containing the type of test executed, the place, the registration method, the number of target users considered, the scripts and environment, the data collected, the results obtained, list of usability issues identified, including category and priority of the problems found.

\section{References}

1. Ballard, B.: Designing the Mobile User Experience. Little Springs Design, Inc., USA. John Wiley \& Sons, Ltd, West Sussex (2007)

2. Tambascia, C., Menezes, E.M.: Aspectos relacionados à Usabilidade. PD.30.13.22A.0001ART19-AA. Technical report (2010)

3. Apple Inc. iPhone Human Interface Guidelines. Human Interface Guidelines. All rights reserved (2010)

4. BlackBerry Smartphones. Version: 2.3. UI Guidelines (2009)

5. Gong, J., Tarasewich, P.: Guidelines for handheld mobile device interface design. College of Computer and Information Science, Northeastern University, Boston (2004)

6. Shneiderman, B.: Designing the User Interface - Strategies for Effective Human-Computer Interaction. Addison-Wesley, Reading (1998) 